\title{
Dinámica del heleoplancton en relación a las fluctuaciones hidrológicas en aiguamolls de l'Empordà (NE de la península lbérica). Propuesta de un método sencillo de monitorización basado en la abundancia de grupos taxonómicos
}

\author{
Ramon Moreno-Amich, Xavier D. Quintana, Lluís Suñer, Rosa Trobajo y Stephanie Gascón. \\ Instituto de Ecología Acuática y Depto. de Ciencias Ambientales. Universidad de Girona. Girona.
}

\section{RESUMEN}

De septiembre de 1996 a agosto de 1997 se llevó un seguimiento de la calidad ecológica del agua del Parque Natural de "Aiguamolls de l'Empordá". El objetivo de este estudio es diseñar una metodología simple para detectar inmediatamente cambios en la calidad del agua debidos a actividades antrópicas como la regulación del caudal por el Parque, entradas de nutrientes procedentes de una planta de tratamiento de aguas residuales próxima, o debidas al uso de fertilizantes agrícolas. El método propuesto se basa en el análisis de la abundancia de grupos taxonómicos característicos de invertebrados acuáticos (heleoplancton). La simplicidad de la metodología está asegurada debido al uso de grupos taxonómicos que son más fáciles de reconocer que las especies. Se ha modelizado el funcionamiento de los sistemas acuáticos por medio del Análisis de Correspondencias entre muestras y taxones. Los resultados muestran cinco situaciones ambientales con unas estructuras de comunidad regulares caracterizadas por la dominancia de un grupo taxonómico: cladóceros, ostrácodos, calanoides, ciclopoides y harpacticoides. Las dinámicas de lagunas de aguas dulce y salobre pueden ser modelizadas como desplazamientos entre las situaciones mencionadas. Mientras que la "situación calanoides" y la "situación harpacticoides" ocurren principalmente en lagunas de aguas salobres, la "situación cladóceros" ocurre principalmente en las de agua dulce. Los cuatro ejes principales identificados son la tasa de renovación de los nutrientes (primer eje), hipertrofia (segundo eje), grado de mineralización de la materia orgánica (tercer eje) y eutrofia (cuarto eje). Se validó el uso de grupos taxonómicos por comparación con el modelo obtenido a través de las especies. Concluimos que, en un sistema tan variable como éste, únicamente la persitencia de situaciones de eutrofización o hipertrofización debe ser considerada como indicador de un bajo valor de calidad ecológica del agua.

Palabras clave: Heleoplancton, estructura de comunidad, humedales mediterráneos, análisis multivariable, seguimiento.

\begin{abstract}
Monitoring of the "ecological water quality" in "Aiguamolls de l'Empordà" Natural Park was carried out between September 1996 and August 1997. The aim of this sampling programme was to design a simple method for rapid detect changes in water quality due to human activity. These include flow regulation by the Park management, nutrient entries from effluents of a nearby wastewater treatment plant or agricultural fertilising. The proposed method is based on the analysis of the abundance of characteristic taxonomic groups of aquatic invertebrates (heleoplankton). The simplicity of the method is ensured by the use of large taxa which are easier to recognise than species. The functioning of aquatic systems has been modelled by means of correspondence analysis between samples and taxa. Results can be summerized in five environmental conditions with a regular community structure. Dominated by one taxon: cladocerans, ostracods, calanoids, cyclopoids and harpacticoids. The dynamics of both freshwater and brackish lagoons can be modelled as displacements between these five groups of environ-
\end{abstract}

Limnetica 16: 17-31 (1999)

(C) Asociación Española de Limnología, Madrid. Spain. ISSN: 0213-8409. 
mental conditions. Nevertheless, the "calanoids situation" and the "harpacticoids situation" occur mainly in brackish lagoons, whereas the "cladocerans situation" occurs mainly in freshwater. The four principal axes of data variation have been respectively identified as nutrient turnover rate, hypertrophy, degree of mineralization of the organic matter and eutrophy. The use of these taxa has been validated by comparison with a model obtained from the species. We conclude that in a highly fluctuating system such as the one here, only persistent situations of eutrophy or hypertrophy must be equated to low "ecological water quality".

Keywords: Heleoplankton, community structure, Mediterranean wetlands, multivariate analysis, monitoring.

\section{INTRODUCCIÓN}

La zona conocida como Aiguamolls de l'Empordà ocupa el litoral de la Bahía de Roses, en el límite norte de la costa mediterránea ibérica. Se trata del frente de una llanura deltaica, originada por los aportes de los ríos Muga y Fluvià (Bach, 1990) y está formado por un conjunto de lagunas costeras, marismas mediterráneas (no influidas por la marea) y zonas inundables (Fig. 1).

En 1984 se creó el Parque Natural de Aiguamolls de l'Empordà (en adelante, PNAE). Toda la superficie del PNAE, especialmente el área sur, es muy rica en aguas subterráneas y sus acuíferos se utilizan para abastecer de agua potable las poblaciones de la zona. En estas poblaciones el turismo y la agricultura representan las principales actividades económicas y este hecho ha sometido a estas zonas húmedas a una importante degradación durante las últimas décadas. A pesar de ello el PNAE conserva todavía un reconocido interés biológico (Sargatal \& Fèlix, 1989; Gosálbez et al., 1994).

Desde su creación la gestión del PNAE ha ido encaminada a "devolver a estas zonas húmedas su antigua extensión y productividad" (Sargatal, 1989), compensando las considerables pérdidas por desecación que ha sufrido la zona durante las últimas décadas. El objetivo principal hasta la actualidad ha sido incrementar las poblaciones de aves tanto sedentarias como visitantes, aumentando la disponibilidad de hábitats acuáticos para las aves mediante el incremento de la superficie inundada. En este sentido se construyó una laguna artificial (Estany del Cortalet) alimentada con agua procedente del Rec Corredor.

De todas las actuaciones quizás la más remarcable por sus consecuencias ha sido la instalación de una compuerta en el canal de desagüe Massona-Rec Sirvent, que entró en funcionamiento en 1990. Con la compuerta se puede ahora retener dentro de la reserva el agua dulce superficial que circula por el Rec Corredor, hasta alcanzar un nivel medio de agua de $0.8 \mathrm{~m}$ sobre el nivel del

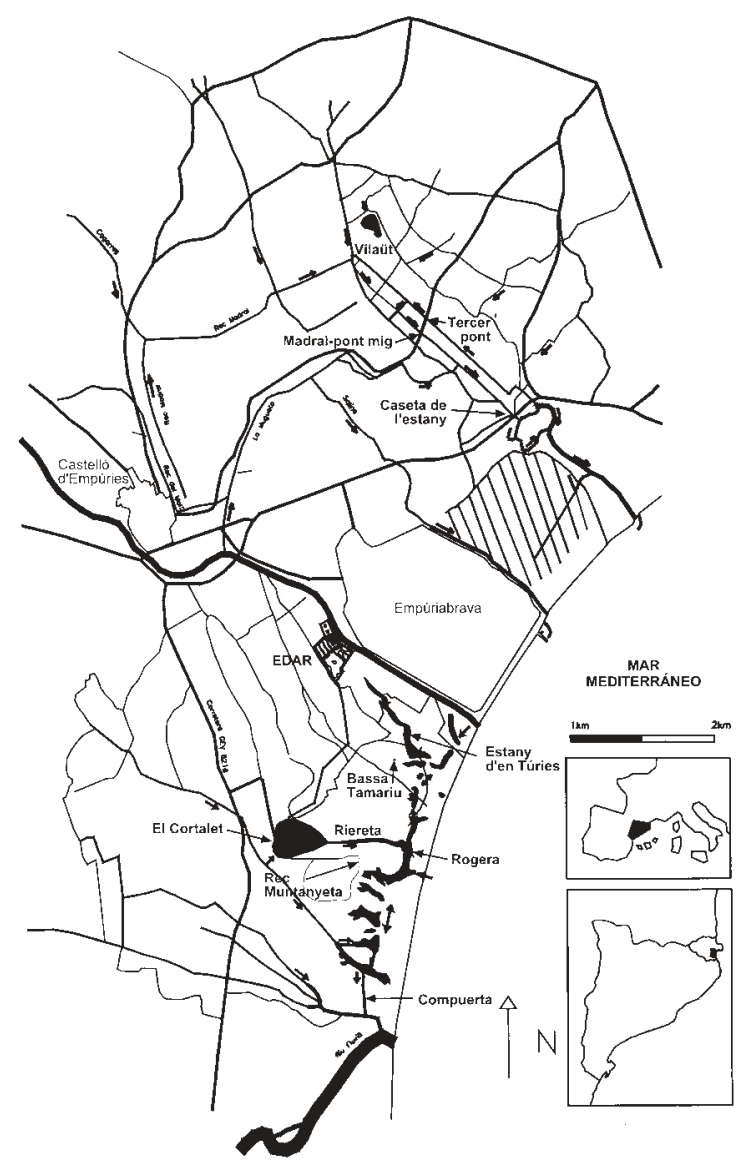

Figura 1. Mapa de situación de los puntos muestreados. Sampling sites location. 
mar durante los períodos de inundación, y ha permitido aumentar considerablemente la superficie inundada (antes, en inviernos normales, era de 37 ha y ahora supera las $200 \mathrm{ha}$ ).

Numerosos estudios destacan la fuerte incidencia que representa la regulación de flujos sobre los ecosistemas costeros mediterráneos. Generalmente, esta incidencia se manifiesta en un importante incremento de la eutrofización (Aguesse \& Marazanof, 1965; López, 1983; Comín, 1984; Miracle, 1984; Pretus, 1985; Ros, 1987; Amanieu et al., 1989; Lasserre, 1989; Quintana \& Comín, 1989; Herteaux, 1992). En nuestro caso se dispone de estudios realizados en el momento de la instalación de la compuerta, que demuestran los cambios en la calidad del agua y en la composición de las comunidades acuáticas planctónicas causadas por la puesta en marcha de dicha compuerta (Quintana, 1995; Quintana et al., 1998a; Quintana et al., 1998b). Además, desde 1996 venimos realizando una monitorización de la calidad ecológica de las aguas del PNAE, a partir de la cual se ha observado un aumento del tiempo de permanencia del agua dulce y de la superficie afectada por la inundación. También la regulación de flujos ha causado un aumento paulatino del grado de eutrofia (Moreno-Amich et al., 1998), debido a la mayor aportación de nutrientes.

Por otro lado, en el territorio del PNAE se ha construido una depuradora de aguas residuales (EDAR) de tipo mixto, con un tratamiento mediante fangos activados con aireación prolongada sin decantación primaria, seguido por un sistema de lagunaje con una primera laguna de sedimentación, donde los fangos se mineralizan, y una segunda laguna de afilamiento, donde se produce la última fase de depuración y desinfección de forma natural. Posteriormente se le ha añadido un sistema de tratamiento adicional del agua depurada mediante un sistema de humedales construidos (SHC), con el objetivo de reducir los niveles de nutrientes del efluente final de la EDAR. Se espera que el efluente de la EDAR sea apto para la creación y restauración de zonas húmedas en el PNAE sin que su utilización suponga un incremento en la eutrofización de la marisma.
En relación a esta actuación se ha iniciado un programa de vigilancia ambiental para constatar que el diseño y funcionamiento del SHC son correctos, y no se produce eutrofización.

El objetivo del presente estudio es diseñar un sistema sencillo de monitorización de la calidad de las aguas que permita detectar con inmediatez el efecto de la regulación de flujos del Parque Natural, de la difusión de nutrientes desde la EDAR, y otras acciones antrópicas que afecten a la calidad de las aguas que circulan por los Aiguamolls de l'Empordà. Se parte de la consideración de que los cambios en la calidad del agua afectarán más rápidamente al plancton que al bentos. Por otra parte, la facilidad de muestreo del zooplancton y de la determinación taxonómica a nivel de grandes grupos, asegura la sencillez del sistema de monitorización. Además, se pretende que el método sea válido tanto para las lagunas de agua dulce como para las lagunas costeras salobres.

Este objetivo general se desglosa en dos objetivos de investigación: 1) Modelizar el funcionamiento de los sistemas acuáticos como una sucesión de situaciones ambientales (caracterizadas por valores regulares de las características físicas y químicas del agua), que sean reconocibles por la dominancia de los principales grupos de invertebrados (heleoplancton); y 2) Relacionar las situaciones ambientales antes mencionadas con la calidad de las aguas según los propósitos de gestión ambiental, básicamente evaluar la eutrofización.

\section{MATERIAL Y MÉTODOS}

\section{Muestreo de invertebrados acuáticos}

Con el objeto de desarrollar un método sencillo de monitorización, se ha restringido el muestreo a los organismos de talla observable a 50 X. Así pues, la captura de invertebrados acuáticos se ha realizado con salabre de $20 \mathrm{~cm}$ de diámetro y red de $250 \mu \mathrm{m}$ de luz de malla. El método consiste en hundir el salabre en el agua y levantarlo para pes- 


\section{Lagunas costeras salobres}
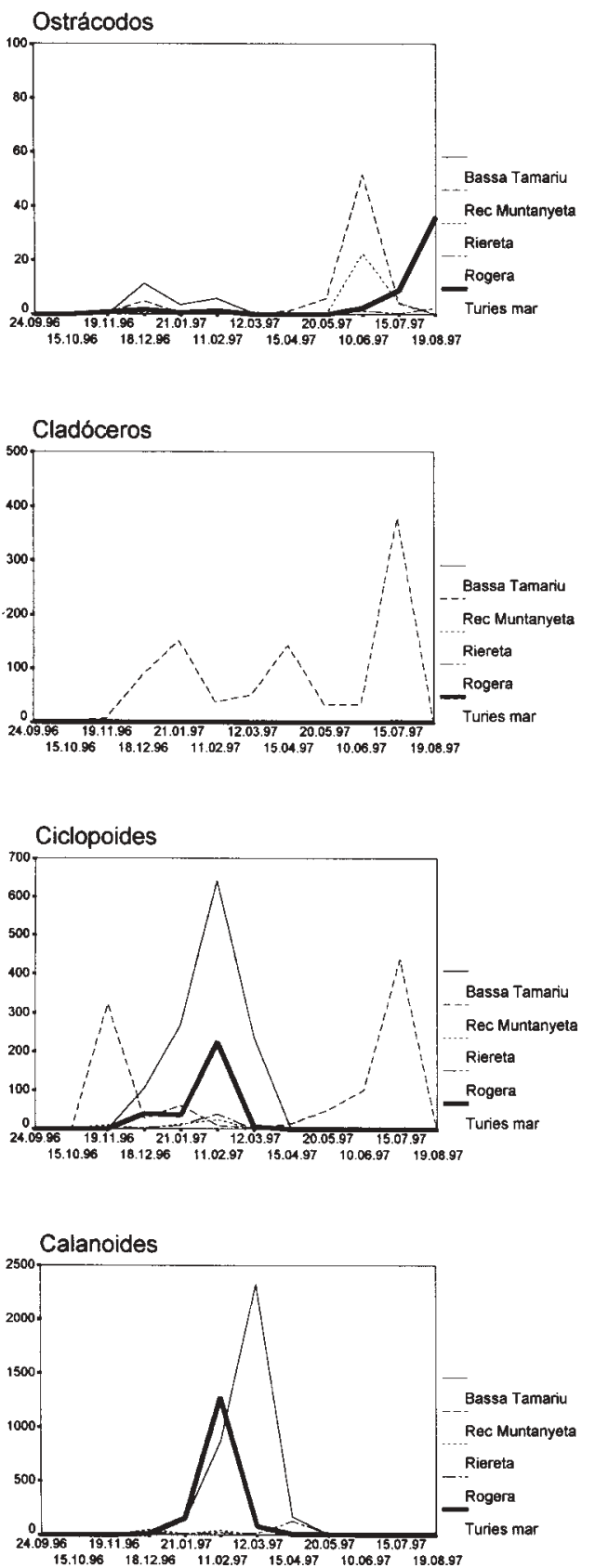

\section{Lagunas de agua dulce}
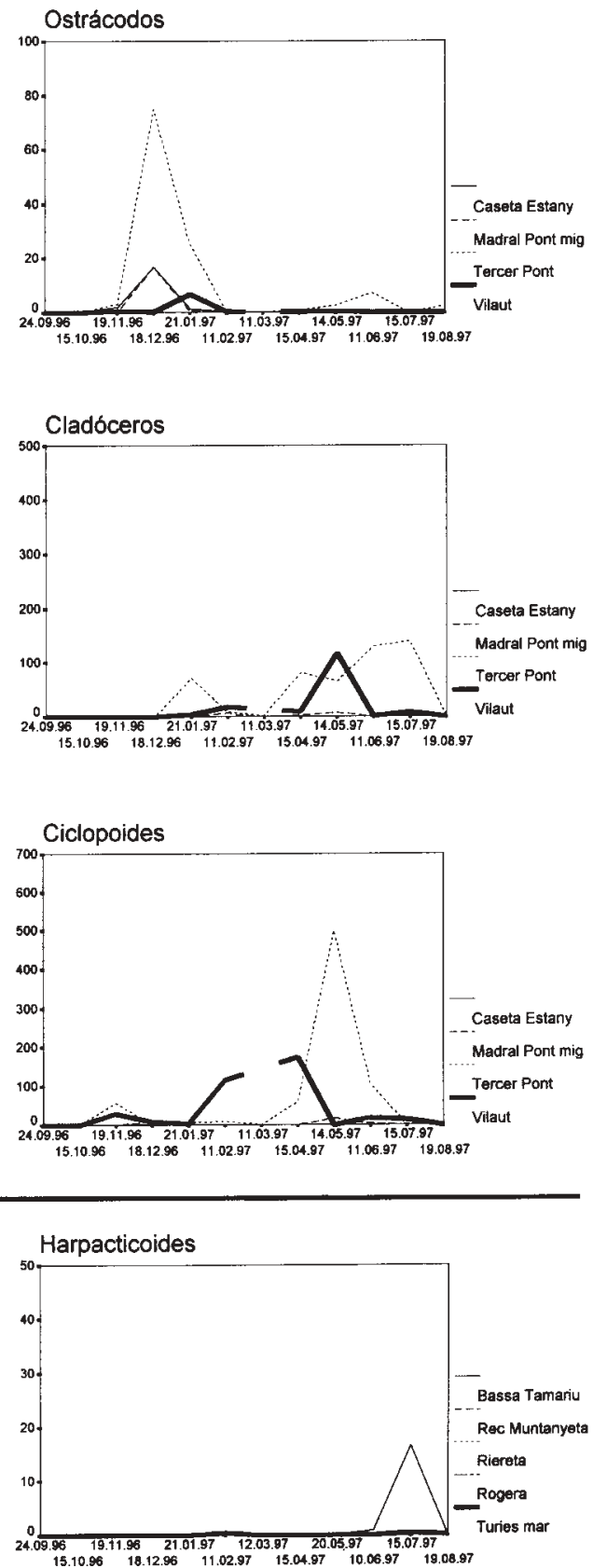

Figura 2. Variación temporal de los grupos de invertebrados acuáticos más abundantes en las lagunas costeras salobres y en los sistemas de agua dulce. Seasonality of the most abundant groups of aquatic invertebrates in coastal lagoons and in freshwater systems. 
car los invertebrados que se encuentran en la columna de agua superior. La captura se repite varias veces (generalmente 20) para aumentar el número de individuos capturados. La abundancia viene referida al número de individuos por salabre (Captura Por Unidad de Esfuerzo). Los organismos capturados se han fijado con formol al $4 \%$ para su posterior recuento y determinación a la lupa binocular y/o al microscopio. Las CPUE no dan una estima precisa de la densidad absoluta, pero permiten la comparación entre fechas y entre abundancias relativas de las especies.

Los muestreos se realizaron de setiembre-1996 a agosto-1997 con periodicidad mensual en 5 puntos correspondientes al sector sur, constituido por lagunas costeras salobres (Rogera, Rec Muntanyeta, Riereta, Bassa Tamariu, y Estany d'en Túries), y 4 puntos correspondientes al sector norte que incluye sistemas acuáticos de agua dulce (Caseta de l'Estany, Madral-pont mig, Tercer pont, y Vilaüt). En ambos sectores se observan amplias variaciones de salinidad (Moreno-Amich et al., 1998).

\section{Análisis de datos}

La dinámica de los sistemas acuáticos estudiados se ha analizado mediante el Análisis de Correspondencias. La similitud entre la dinámica de los diferentes sistemas se ha establecido mediante un Análisis de Cluster (algoritmo UPGMA) a partir de la matriz de distancias entre puntos de muestreo, calculadas como distancia euclídea.

La validación del modelo basado en grupos taxonómicos se ha realizado analizando la posición que correspondería a las especies en este sistema de coordenadas (Jambu, 1991).

\section{RESULTADOS}

\section{Composición específica}

En la composición de invertebrados capturados con salabre dominan principalmente los crustá- ceos. Los cladóceros y los tres grupos de copépodos de vida libre (calanoides, ciclopoides y harpacticoides) suman muy frecuentemente más del $90 \%$ de los individuos. En determinadas ocasiones pueden ser también abundantes otros grupos de crustáceos, como los ostrácodos, los anfípodos o los misidáceos, y de insectos, como los quironómidos, los heterópteros, los coleópteros y los efemerópteros. En general, la estructura de la comunidad es característica de ambientes lagunares costeros y se acerca a la observada en otros ambientes similares de la costa mediterránea (Aguese \& Marazanof, 1965; Bigot \& Marazanov, 1965; Hervé \& Bruslé, 1981; Sabater, 1981; Menéndez \& Comín, 1986; Carrillo et al., 1987; Pretus, 1989; Quintana \& Comín, 1989; López et al., 1991).

A pesar de que la mayor parte de las especies importantes se encuentran indistintamente en los dos sectores (lagunas de agua dulce y lagunas costeras salobres), existen diferencias importantes en cuanto a la abundancia relativa de las principales especies.

En las lagunas de agua dulce dominan los ciclopoides y los cladóceros (Fig. 2). Los cladóceros están muy diversificados en estas lagunas y se han identificado hasta 22 especies diferentes. Bosmina longirostris, Simocephalus vetulus, Scapholeberis mucronata, Daphnia magna, Chydorus sphaericus, Alona rectangula, Tretocephala ambigua, Pleuroxus denticulatus y Daphnia pulicaria son las especies más abundantes de este grupo. Los ciclopoides son más abundantes que los cladóceros, pero están representados per un número muy inferior de especies. Acanthocyclops robustus, Cyclops sp., Thermocyclops dybowskii, Eucyclops serrulatus, Diacyclops bicuspidatus odessanus y Macrocyclops albidus son las especies más representativas. Calanoides (como Calanipeda aquaedulcis o Mixodiaptomus kupelwieseri) y harpacticoides (como Canthocamptus staphylinus, Nitocra spinipes o Phyllognathopus viguieri) son bastante escasos en estas lagunas.

Los ostrácodos poden también aparecer en determinadas condiciones con poblaciones bastante numerosas. Las especies más abundantes 
Table 1. Solución finalmente aceptada del Análisis de Correspondencias. Se indican los taxones con mayor contribución (>0.100) a la inercia de cada dimensión (en negrita) o con mayor coordenada. Final accepted solution of correspondence analysis. Taxa with greater contribution (>0.100) to the inertia of dimensions (in bold) or with high coordinate are presented.

\begin{tabular}{|c|c|c|c|c|}
\hline Dimensión & $\begin{array}{l}\text { Proporción explicada } \\
\text { (acumulada) }\end{array}$ & $\begin{array}{c}\text { Taxones } \\
\text { explicativos }\end{array}$ & $\begin{array}{c}\text { Contribución a la } \\
\text { inercia de la dimensión }\end{array}$ & $\begin{array}{c}\text { Coordenada en } \\
\text { la dimensión }\end{array}$ \\
\hline \multirow[t]{2}{*}{1} & 0.29 & Cladóceros & 0.449 & -1.645 \\
\hline & $(0.29)$ & Calanoides & 0.410 & 0.855 \\
\hline \multirow[t]{2}{*}{2} & 0.21 & Harpacticoides & 0.699 & 15.517 \\
\hline & $(0.50)$ & Ostràcodes & 0.177 & 2.076 \\
\hline \multirow[t]{2}{*}{3} & 0.20 & Ostrácodos & 0.629 & -3.836 \\
\hline & $(0.70)$ & Harpacticoides & 0.253 & 9.159 \\
\hline \multirow[t]{3}{*}{4} & 0.13 & Ciclopoides & 0.589 & -1.005 \\
\hline & $(0.83)$ & Cladóceros & 0.293 & 1.098 \\
\hline & & Calanoides & 0.108 & 0.364 \\
\hline
\end{tabular}

son Eucypris virens, Heterocypris incongruens, Cypridopsis vidua y Potamocypris variegata. Con abundancias inferiores se pueden encontrar diversas especies de insectos, especialmente larvas de quironómidos y heterópteros. Entre los primeros, muy diversificados (34 taxones) predominan los géneros Thienemannia, Orthocladius, Corynoneura y Cricotopus; entre los segundos, Sigara lateralis, Notonecta sp. y Sigara dorsalis.

En las lagunas costeras salobres los calanoides son los organismos más abundantes (Fig. 2), especialmente Calanipeda aquae-dulcis y Eurytemora velox. También se encuentran altas densidades de ciclopoides, principalmente de Diacyclops bicuspidatus odessanus, Cyclops sp. y Acanthocyclops robustus. Son mucho menos abundantes los cladóceros, aunque Daphnia pulicaria puede formar poblaciones densas en las partes más dulces, como en "rec Muntanyeta", un canal de desagüe de agua dulce.

Destaca en este sector la mayor importancia de los harpacticoides, entre los que dominan las especies Cletocamptus confluens y Nitocra lacustris. La presencia de harpacticoides (básicamente Cletocamptus confluens) en "Bassa Tamariu", puede relacionarse con la ocurrencia de episodios de hipertropfia, es decir, episodios prolongados de falta de oxigeno a causa de una excesiva concentración de materia orgánica, que favorecen a las especies adaptadas a tolerar condiciones de anoxia. Entre los ostrácodos destacan Cyprideis torosa, Sarscypridopsis aculeata y Loxoconcha elliptica. Otros crustáceos, como el anfípodo Gammarus aequicauda, el isópodo Sphaeroma hookeri y el misidáceo Mesopo dopsys slabberi son también mucho más abundantes en las lagunas salobres que en las dulces.

\section{Dinámica del sistema}

El análisis de la dinámica del sistema se ha abordado mediante el Análisis de Correspondencias (AC), restringiéndolo en un segundo paso a aquellos grupos taxonómicos con una mayor contribución a la inercia de las dimensiones del AC. Así pues los grupos considerados finalmente son: oligoquetos cladóceros, ostrácodos, calanoides, ciclopoides, harpacticoides, anfípodos y quironómidos.

En la solución final del AC se han aceptado 4 dimensiones (Tabla 1), que explican el $83 \%$ de la variabilidad de las muestras. En la figura 1 se representan conjuntamente las muestras y los 
taxones en el espacio de los factores (1, 2, 3 y 4), mediante tres proyecciones bidimensionales.

La interpretación de los ejes se ha basado en la identificación de los taxones con mayor peso explicativo para cada dimensión (Tabla 1), y las correlaciones de éstos con las características físicas y químicas del agua (Tabla 2). Así, la dimensión 1 se ha interpretado como un gradiente relacionado con la tasa de renovación de nutrientes, que opone las situaciones de confinamiento (con una baja tasa de renovación) a las situaciones de alta tasa de aporte o reciclado de nutrientes. La dimensión 2 corresponde a un gradiente de importancia de la vía detrítica relacionado con los episodios de hipertrofia por pulsos de nutrientes o con la concentración de materia orgánica durante el proceso de desecación de las lagunas. La dimensión 3 se relaciona con el grado de mineralización de la materia orgánica. Finalmente, la dimensión 4 está relacionada con la disponibilidad de nutrientes y se interpreta como un gradiente de eutrofia.

A partir del Análisis de Correspondencias (Fig. 3 ), se han identificado 5 situaciones que se caracterizan por la dominancia de sendos grupos taxonómicos:

- Situación de Calanoides. Situación de confinamiento, con tasa de renovación del agua prácticamente nula y sin aporte externo de nutrientes. Se trata de una situación de oligotrofia relativa en que toda la producción

Table 2. Coeficientes de correlación de Spearman $\left(\mathrm{r}_{\mathrm{s}}\right.$ ) entre las dimensiones del Análisis de Correspondencias y las características físicas y químicas del agua. En negrita se indican las correlaciones significativas $(\mathrm{p}<0.05)$. Spearman correlation coefficients $\left(r_{s}\right)$ between the four principal axes of the correspondence analysis and physico-chemical variables. Significant correlations are marked in bold ( $p<0.05)$.

\begin{tabular}{|c|c|c|c|c|c|c|c|c|}
\hline \multirow[t]{3}{*}{ Característica } & \multicolumn{8}{|c|}{ Coef. correlación de Spearman } \\
\hline & \multicolumn{2}{|c|}{ Dim1 } & \multicolumn{2}{|c|}{ Dim2 } & \multicolumn{2}{|c|}{ Dim3 } & \multicolumn{2}{|c|}{ Dim4 } \\
\hline & $\mathbf{r}_{\mathrm{s}}$ & $\mathbf{p}$ & $\mathbf{r}_{\mathrm{s}}$ & $\mathbf{p}$ & $\mathbf{r}_{\mathrm{s}}$ & $\mathbf{p}$ & $\mathbf{r}_{\mathrm{s}}$ & $\mathbf{p}$ \\
\hline Nivel de agua & 0.2508 & 0.023 & -0.3993 & 0.000 & 0.2609 & 0.018 & -0.1546 & 0.166 \\
\hline Caudal & 0.0962 & 0.687 & 0.6480 & 0.002 & -0.6038 & 0.005 & 0.2349 & 0.319 \\
\hline $\begin{array}{l}\text { Temperatura } \\
\text { superficie }\end{array}$ & 0.0876 & 0.443 & 0.0964 & 0.398 & -0.0228 & 0.842 & 0.1619 & 0.154 \\
\hline $\begin{array}{l}\text { Temperatura } \\
\text { fondo }\end{array}$ & 0.3995 & 0.059 & 0.3036 & 0.159 & -0.3159 & 0.142 & 0.2067 & 0.344 \\
\hline $\begin{array}{l}\text { Conductividad } \\
\text { superficie }\end{array}$ & 0.5670 & 0.000 & -0.0214 & 0.851 & 0.0933 & 0.413 & 0.0786 & 0.491 \\
\hline $\begin{array}{l}\text { Conductividad } \\
\text { fondo }\end{array}$ & 0.3957 & 0.056 & 0.0739 & 0.731 & -0.0922 & 0.668 & -0.4984 & 0.013 \\
\hline pH superficie & 0.2571 & 0.023 & -0.2904 & 0.010 & 0.1336 & 0.243 & 0.1517 & 0.185 \\
\hline $\mathrm{pH}$ fondo & 0.2685 & 0.281 & 0.4770 & 0.045 & -0.4471 & 0.063 & -0.0671 & 0.791 \\
\hline $\mathrm{O}_{2}$ & 0.1120 & 0.570 & -0.3736 & 0.050 & -0.0408 & 0.837 & 0.1282 & 0.516 \\
\hline $\begin{array}{l}\text { Saturación } \mathrm{O}_{2} \\
\text { superficie }\end{array}$ & 0.4168 & 0.000 & -0.1131 & 0.324 & 0.0508 & 0.659 & 0.1345 & 0.241 \\
\hline $\begin{array}{l}\text { Saturación } \mathrm{O}_{2} \\
\text { fondo }\end{array}$ & 0.1199 & 0.636 & 0.1891 & 0.452 & -0.1840 & 0.465 & -0.0506 & 0.842 \\
\hline Clorofila & 0.3011 & 0.008 & -0.2777 & 0.014 & 0.2731 & 0.016 & -0.2605 & 0.022 \\
\hline Fosfato & -0.3904 & 0.000 & -0.0199 & 0.862 & 0.0848 & 0.457 & -0.1170 & 0.304 \\
\hline Amonio & -0.2544 & 0.025 & 0.3453 & 0.002 & -0.2589 & 0.022 & -0.0705 & 0.540 \\
\hline Nitrito & 0.1107 & 0.332 & 0.2664 & 0.018 & -0.3362 & 0.002 & -0.1661 & 0.144 \\
\hline Nitrato & -0.1721 & 0.129 & 0.0282 & 0.805 & -0.1994 & 0.078 & -0.0583 & 0.610 \\
\hline Materia orgánica & 0.5694 & 0.000 & -0.1011 & 0.415 & 0.1265 & 0.308 & -0.1008 & 0.417 \\
\hline Alcalinidad & 0.2591 & 0.034 & -0.4189 & 0.000 & 0.3564 & 0.003 & 0.1563 & 0.206 \\
\hline
\end{tabular}



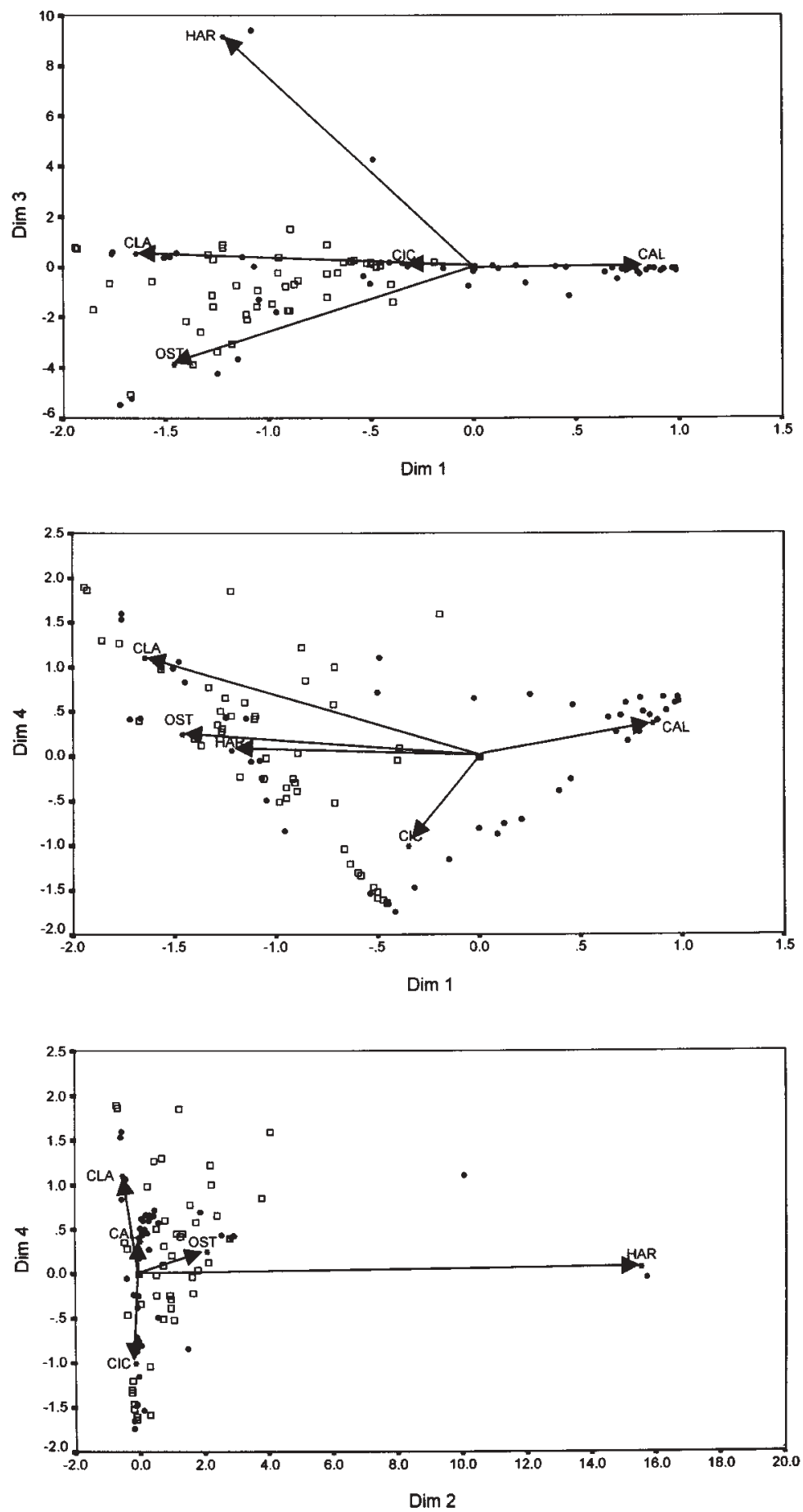

Figura 3. Representación de las muestras y los grupos taxonómicos en el espacio factorial $(1,2,3,4)$ del Análisis de Correspondencias. Representation of samples and taxonomic groups in the factor space $(1,2,3,4)$ of correspondence analysis. 

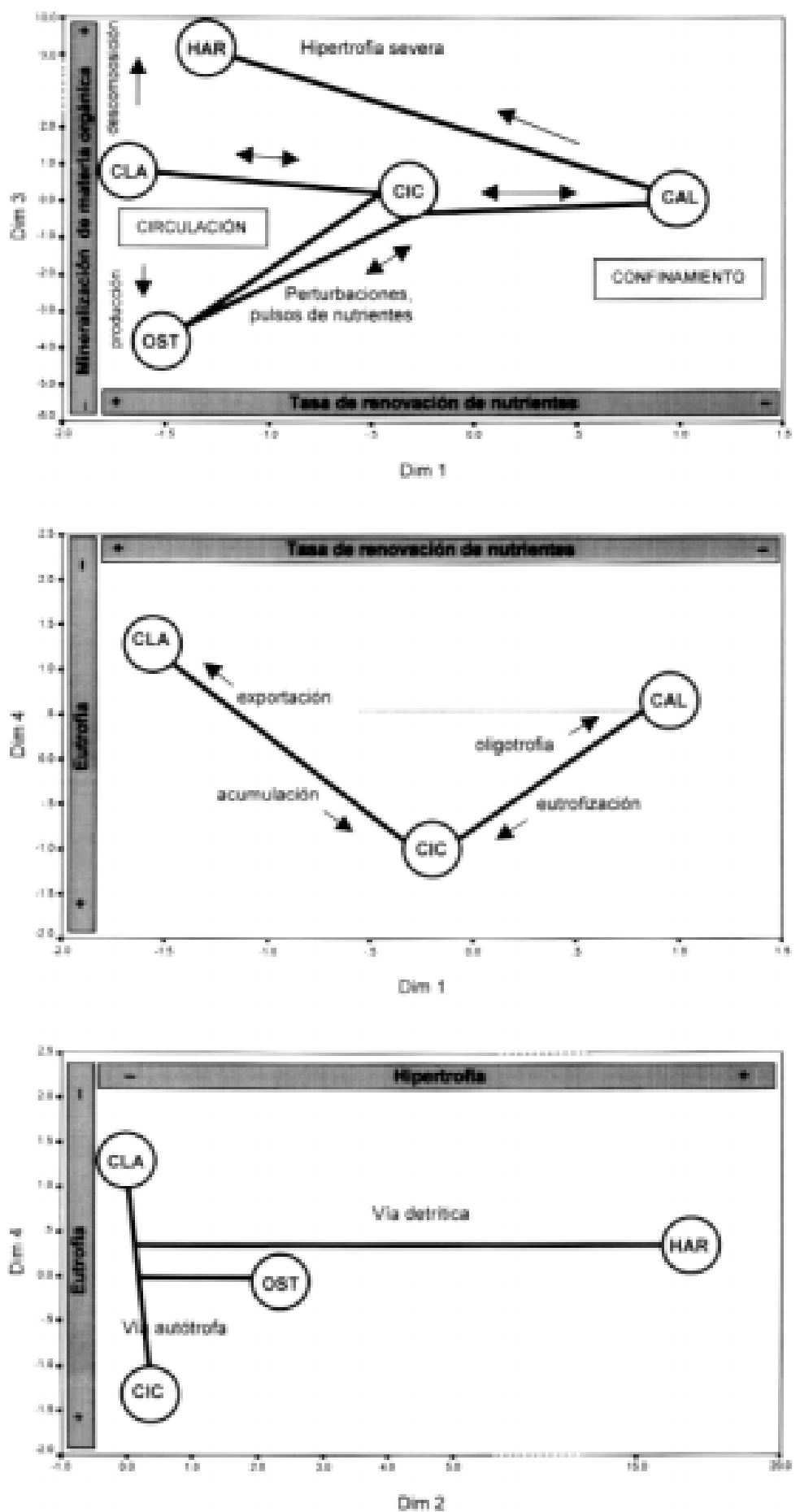

Figura 4. Modelo de la dinámica de los sistemas acuáticos de Aiguamolls de l'Empordà según la sucesión temporal de las situaciones ambientales en el espacio factorial $(1,2,3,4)$ del Análisis de Correspondencias. Model of the dynamics of the aquatic systems of Aiguamolls de l'Empordà based on the occurrence of environmental situations in factor space $(1,2,3,4)$ of the correspondence analysis. 
proviene del reciclaje interno de nutrientes.

- Situación de Cladóceros. Situación donde hay circulación continua de agua (tasa elevada y constante de renovación del agua) y un aporte externo de nutrientes continuo. Debido a la circulación se produce exportación de nutrientes.

- Situación de Ciclopoides. Situación con una tasa baja pero constante de renovación de agua y nutrientes, con acumulación de materia orgánica. Esta situación se da en sistemas lóticos con circulación muy lenta del agua, o en sistemas leníticos con aportes continuos pero de poco caudal.

- Situación de Ostrácodos. Situación de aporte masivo de agua, asociado a perturbaciones (inundación), y por tanto con una tasa muy elevada de renovación del agua pero de corta duración. La inundación comporta un aporte masivo de nutrientes y de materia orgánica alóctona durante un corto período de tiempo.

- Situación de Harpacticoides. Situación de acumulación de materia orgánica, asociada al proceso de desecación de las lagunas, que provocan episodios de hipertrofia severa. Esta situación sólo se presenta en las lagunas más eutróficas.

La dinámica de los sistemas se ha modelizado (Fig. 4) a partir de la sucesión temporal de las situaciones ambientales caracterizadas anteriormente. No todas las situaciones se presentan en todos los lugares. Así, las situaciones de dominancia de calanoides y la de harpacticoides sólo se dan en las lagunas salobres (ver ejemplo en Fig. 5) y la de dominancia de cladóceros sólo en las lagunas de agua dulce (ver ejemplo en Fig. 6).

\section{Funcionamiento de las lagunas costeras salobres}

En las lagunas costeras salobres la situación de dominancia de calanoides corresponde a la situación de oligotrofia habitual en condiciones de confinamiento, cuando se ha producido el estancamiento de las aguas. Esta situación es
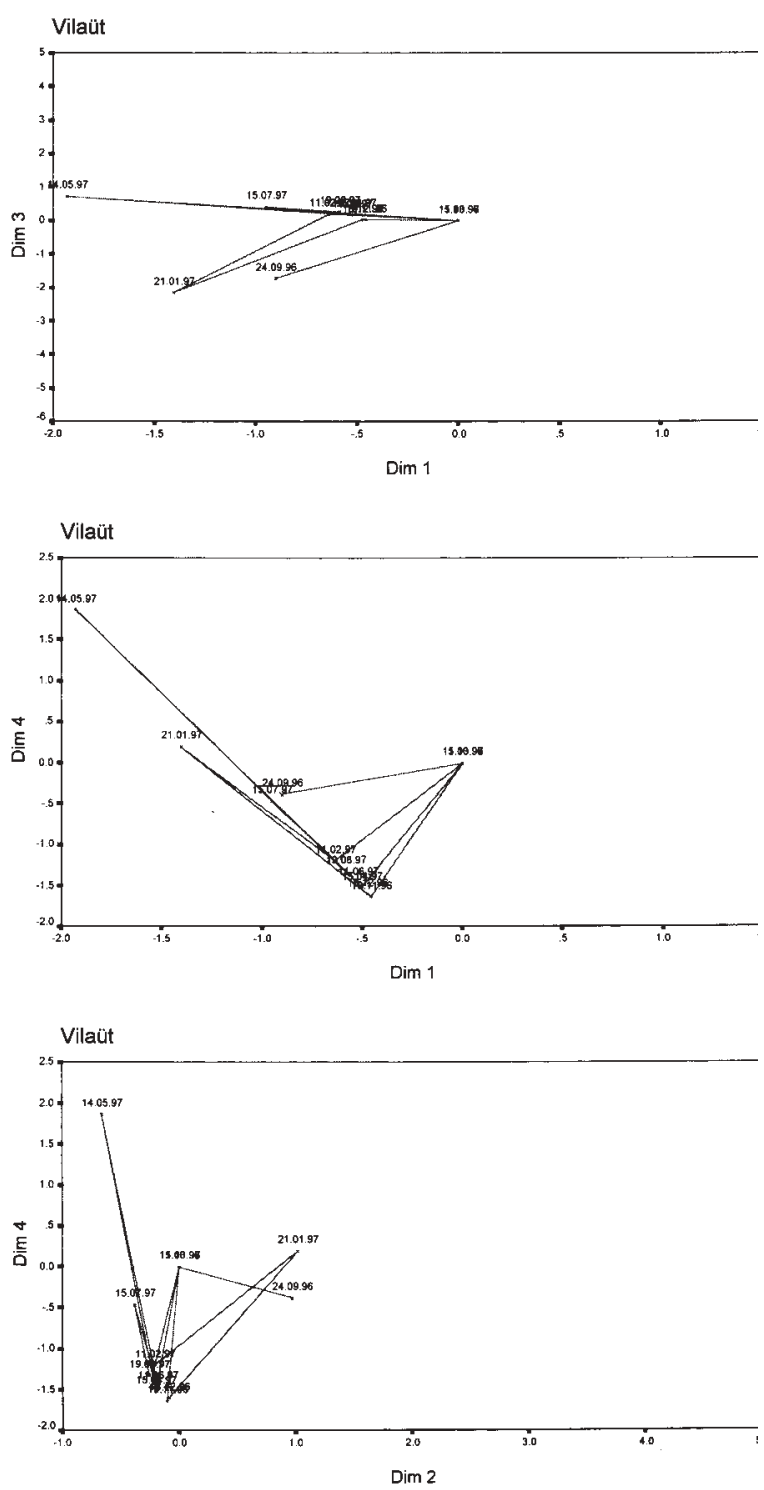

Figura 5. Ejemplo de dinámica de una laguna salobre (La Riereta). Example of dynamics of a brackish lagoon (La Riereta).

alterada por la desecación estival y por las perturbaciones normales en estos ambientes que provocan una entrada de agua con la consiguiente fertilización por la aportación de nutrientes y materia orgánica alóctona.

En el caso de la desecación estival en las lagunas más confinadas (como Bassa Tamariu), la 

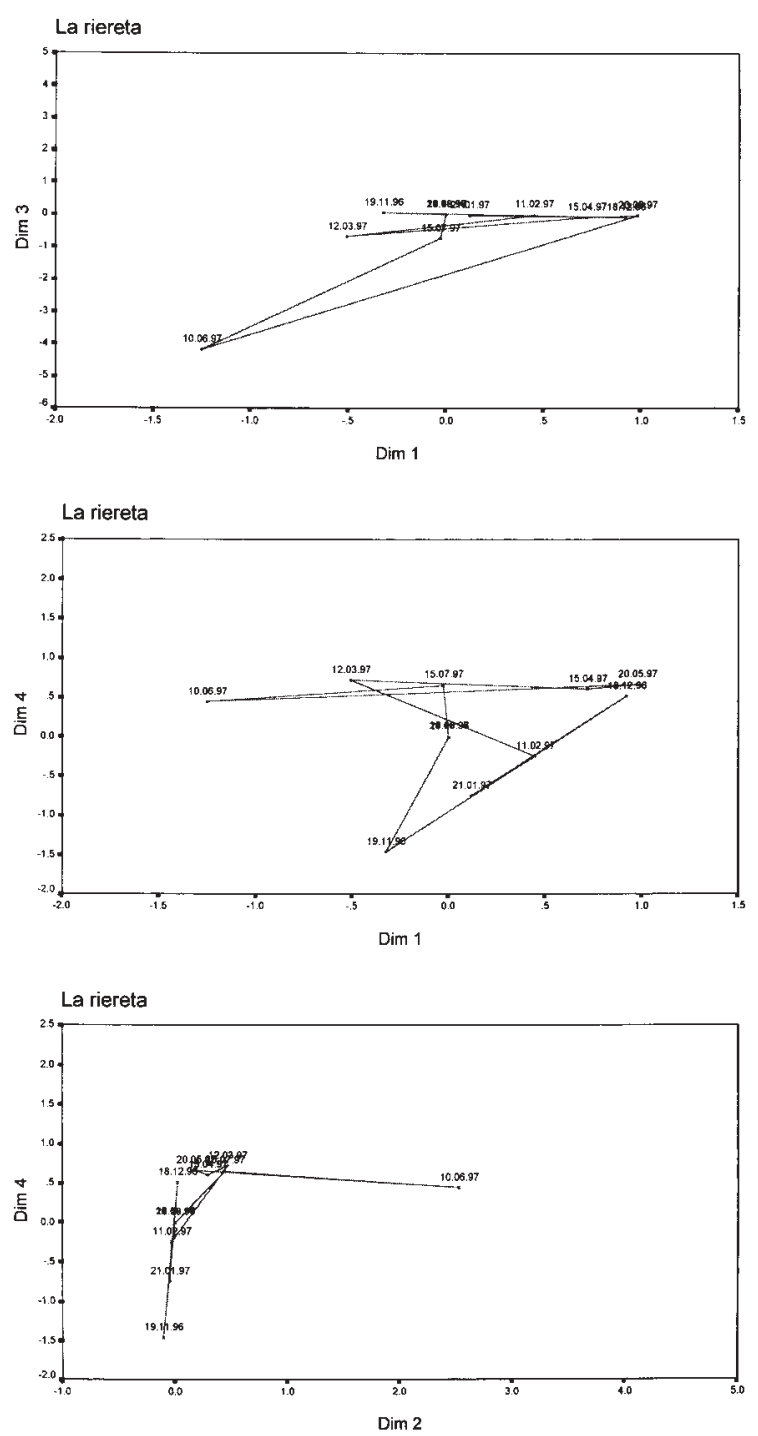

Figura 6. Ejemplo de dinámica de una laguna de agua dulce (Vilaüt). Example of dynamics of a freshwater lagoon (Vilaüt).

progresiva concentración de la materia orgánica acaba produciendo una hipertrofia severa por un exceso de materia orgánica en descomposición y la consiguiente anoxia. Estas condiciones favorecen el desarrollo poblacional de organismos más típicamente bentónicos, detritívoros y más tolerantes a las condiciones de anoxia, como los har- pacticoides. En esta situación, una entrada de agua con la consiguiente aportación de nutrientes tiene un efecto fertilizador adicional que provoca un incremento de materia orgánica muerta que magnifica aún más la situación de hipertrofia.

En el caso de las perturbaciones por entrada de agua, si ésta es puntual (temporales de levante, lluvias) el sistema se desplaza temporalmente hacia la situación de ostrácodos hasta que ha desaparecido el exceso de materia orgánica producida por la fertilización súbita.

En cambio, si la entrada de agua (y la fertilización por entrada de nutrientes) es más paulatina y persistente en el tiempo (caso del cierre de la compuerta) se acaba instaurando una comunidad dominada por ciclopoides, hasta que la fertilización cesa y otra vez se produce el estancamiento y la situación de oligotrofía antes mencionada.

\section{Funcionamiento de las lagunas de agua dulce}

En las lagunas de agua dulce la situación de dominancia de cladóceros corresponde a la situación de circulación de agua, con una elevada tasa de renovación de nutrientes y una exportación de materia orgánica con el curso del agua.

Cuando el caudal disminuye, decrece la tasa de renovación de nutrientes y de materia orgánica alóctona. Este estancamiento relativo conlleva una acumulación de nutrientes que permite el establecimiento de una comunidad dominada por ciclopoides.

Análogamente a como sucede en las lagunas costeras salobres, cuando se produce una fertilización puntual por una aportación externa de nutrientes, el sistema se desplaza hacia la situación de ostrácodos hasta que ha desaparecido el exceso de materia orgánica producida por la fertilización.

\section{Análisis comparativo de la dinámica temporal}

El análisis de cluster agrupa los puntos de muestreo en cuatro grupos relevantes (Fig. 7). Estos grupos, tal como era de esperar, siguen prácticamente 


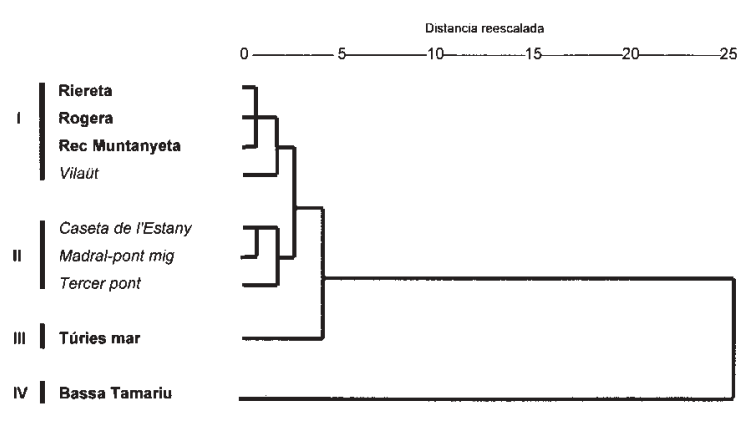

Figura 7. Clasificación según la similitud de la dinámica de los sistemas estudiados. En negrita, lagunas salobres; en cursiva, sistemas de agua dulce. Clustering of systems according to similerities in their dynamics. Brackish lagoons are marked in bold; freshwater systems are in italics.

un patrón de agrupamiento geográfico a excepción del primer grupo. Así el Grupo I contiene los puntos de las lagunas salobres con alguna circulación del agua, más Vilaüt que es una laguna de agua dulce con un alto grado de confinamiento y un cierto nivel de aportaciones de nutrientes. Su similitud con Riereta, Rogera y Rec Muntanyeta, se basa en la frecuencia de la situación de ciclopoides debido a la eutrofia y en presentar desplazamientos hacia la situación de ostrácodos cuando se producen aportaciones puntuales de nutrientes. El Grupo II incluye los sistemas de agua dulce con circulación. El Grupo III incluye la laguna costera salobre "Estany d'en Túries" que presenta confinamiento. Y finalmente el Grupo IV incluye la laguna costera salobre "Bassa Tamariu", también confinada, pero donde se producen episodios de hipertrofia.

\section{DISCUSIÓN}

\section{Validación del modelo basado en grupos taxonómicos}

De acuerdo con los objetivos iniciales se ha desarrollado un modelo de funcionamiento del sistema basado en el reconocimiento de los grupos taxonómicos dominantes (en términos de abundancia), asegurando así la sencillez de la monito- rización que podrá ser realizado por personal no especializado.

El modelo así construido supone que la dominancia de un grupo taxonómico está ligada a la existencia de unas condiciones ambientales determinadas, y que esta relación puede establecerse sean cuales fueren las especies implicadas en cada momento.

Para que el modelo sea válido debe producirse o bien que las especies presentes en la zona de un grupo determinado tengan características poblacionales y ecológicas similares, o bien que la dominancia de un grupo taxonómico siempre esté ligada a una o unas pocas especies que son las más abundantes.

La representación de las especies en el espacio de los factores principales del AC (Fig. 8), sólo se muestran las proyecciones bidimensionales en las que los grupos tienen algún peso explicativo) indica que en general la posición de las especies más abundantes (las que en alguna muestra superan el $10 \%$ de abundancia relativa) coincide en la misma región que el grupo taxonómico correspondiente.

Esto es bien aparente en el caso de los ostrácodos y de los ciclopoides. En lo que se refiere a los calanoides y a los harpacticoides algunas especies aparecen alejadas de la posición del grupo. Sin embargo, se trata de especies siempre poco abundantes (en ninguna muestra superan el $10 \%$ de abundancia relativa) y de baja ocurrencia (sólo en una o muy pocas muestras). Es el caso del calanoide Mixodiaptomus kupelwieseri o del harpacticoide Canthocamptus sthaphylinus. Quizás el caso de los cladóceros es el que necesita más comentario. Las especies más abundantes aparecen claramente en la misma región que el grupo, a excepción de Alona rectangula y Leydigia leydigii. Pero cabe decir que estas especies sólo superan el $10 \%$ de frecuencia relativa en una sola muestra. Las otras especies que aparecen alejadas (Ilyocryptus sordidus, Moina brachiata, Alona guttata y Pleuroxus denticulatus) son especies poco abundantes y de baja ocurrencia.

Así pues de acuerdo con estos resultados y a los algoritmos implicados en al Análisis de Correspondencias (Jambu, 1991) puede considerarse que el uso del modelo basado en grupos taxonómicos es válido puesto que las dominancias de las espe- 
Calanoides

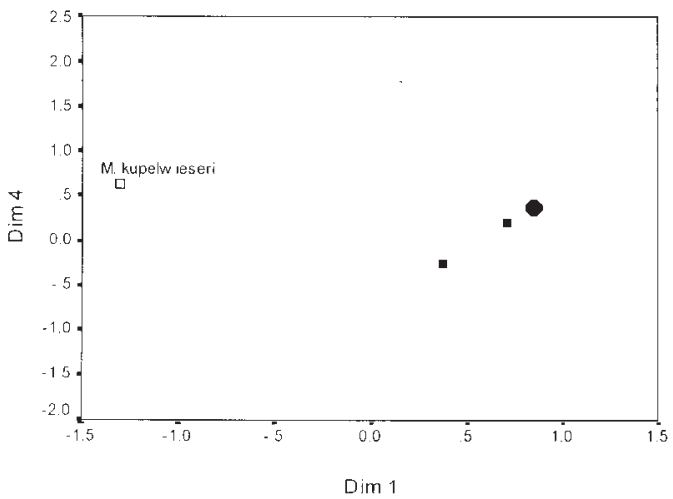

Cladóceros

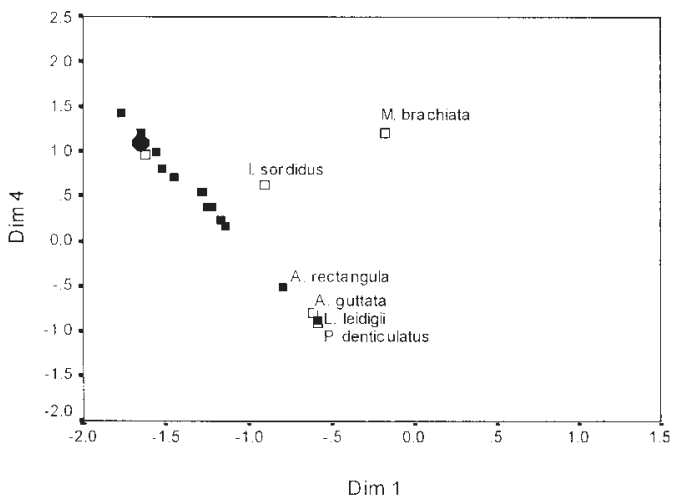

Ciclopoides

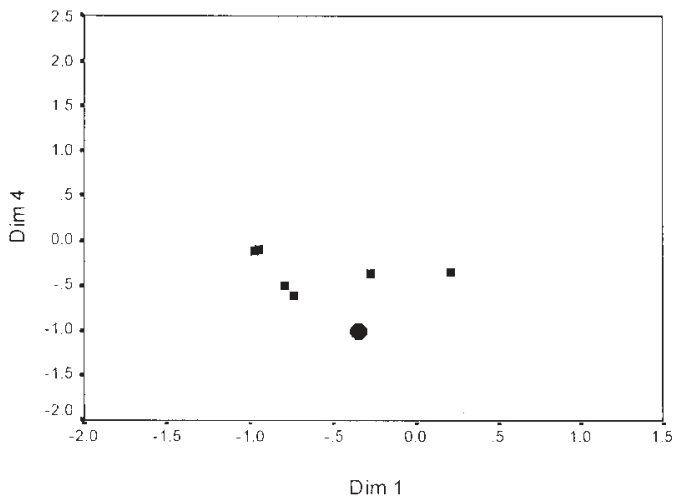

Harpacticoides

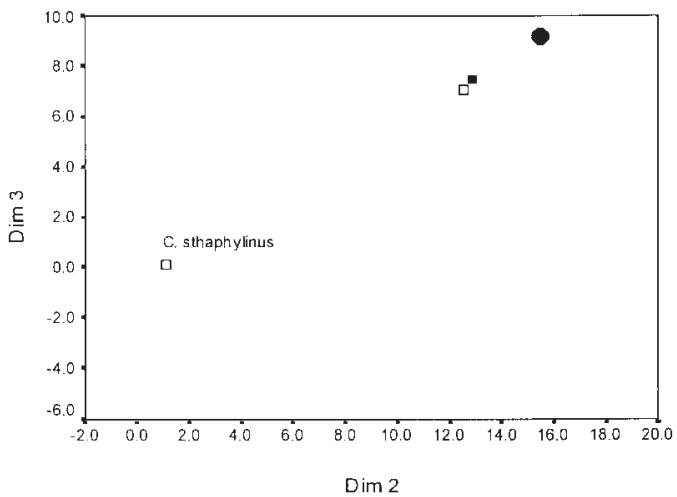

Ostrácodos

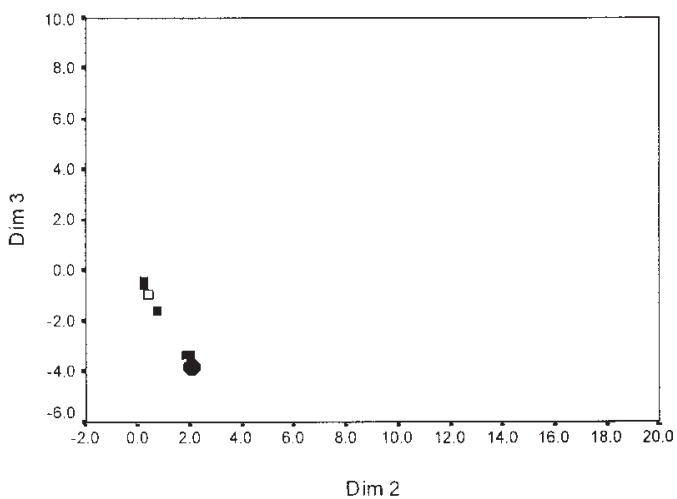

Figura 8. Representación de puntos suplementarios correspondientes a las especies en el espacio factorial (1, 2, 3, 4) del Análisis de

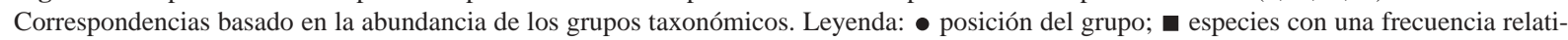
va mayor que $10 \%$ en alguna muestra; $\square$ species con una frecuencia relativa máxima entre el 5\% y el 10\%. Los rótulos corresponden a aquellas especies cuyas coordenadas se apartan de las del grupo taxonómico correspondiente (ver comentario en el texto). Representation of additional points corresponding to species in factor space $(1,2,3,4)$. Correspondence analysis is based on the abundance of taxonomic groups. Legend: - group position; $\square$ species with relative frequency greater than $10 \%$ in at least one sample; $\square$ species with maximum relative frequency between 5 and 10\%. Labelled species are those found far from the taxonomic group position they belong to (see text for details). 
cies que engloba tendirían a dar a las muestras analizadas coordenadas similares a las obtenidas.

\section{Organismos indicadores y estado del sistema}

La utilización de los organismos como indicadores de la calidad de los ecosistemas es corriente en ecología, y su uso se ha desarrollado especialmente en sistemas lóticos (Prat et al., 1983). La composición de una comunidad de organismos no refleja condiciones instantáneas, sino la integración de las características del ambiente sobre cierto tiempo, y por esto revela factores que operan de vez en cuando y que se escapan fácilmente a un análisis, incluso a análisis repetidos (Margalef, 1983). Esta perspectiva parece bastante adecuada en un sistema fluctuante como el de los Aiguamolls de l'Empordá, donde para muchos factores es más relevante su variabilidad que el valor medido en un momento determinado (condicionado por el diseño del muestreo). En el caso de las lagunas costeras salobres, la mayor abundancia o permanencia en el tiempo de especies típicas de aguas eutróficas, como los ciclopoides, o de especies características de situaciones de hipertrofia, corroboran la interpretación que se desprende de los valores de nutrientes inorgánicos (Quintana et al., este volumen, pág. 49).

Tal como se desprende del CA, las dimensiones 1 y 3 describen el funcionamiento del sistema (en términos de tasa de renovación de nutrientes y de concentración de materia orgánica, respectivamente), mientras que las dimensiones 2 y 4 son descriptores del estado trófico del sistema (en términos de hipertrofia y de eutrofia, respectivamente).

Así pues, las dimensiones 2 y 4 nos marcan las pautas para una evaluación de la calidad del agua. Los organismos indicadores asociados a estas dimensiones son los harpacticoides (indicadores de hipertrofia, de exceso de materia orgánica en descomposición) para la dimensión 2 y los ciclopoides (indicadores de eutrofia, de exceso de nutrientes) para la dimensión 4 (Fig. 8).

Desde nuestro punto de vista, tratándose de un sistema fluctuante como el de los Aiguamolls, lo que ha de ser considerado como situación de baja calidad del agua es la persistencia en el tiempo de situaciones de eutrofia o de hipertrofia. La presencia de ciclopoides o harpacticoides ya es en sí misma indicadora de al menos una cierta persistencia (a escala planctónica de unos días o de unas pocas semanas) de las condiciones de eutrofia o de hipertrofia, pero por el carácter fluctuante del sistema a efectos de gestión se ha de considerar como indicador de baja calidad del agua la persistencia de estas situaciones a lo largo de meses.

En este sentido, los dos ejemplos que se aportan corresponden a los casos de peor calidad del agua de lagunas salobres (Riereta, Fig. 5, que alcanza coordenadas más extremas en las dimensiones 2 y 4) y de lagunas de agua dulce (Vilaüt, Fig. 6, donde se puede observar que la mayor parte de las muestras se encuentran en la situación de ciclopoides).

\section{AGRADECIMIENTOS}

Este trabajo ha sido financiado parcialmente por el proyecto CICYT HID96-0916 "Fluctuaciones de alta frecuencia y tendencia del sistema de la marisma, en los "Aiguamolls de l'Empordà". Influencia de la gestión hídrica, efecto del control de mosquitos y viabilidad de la reinundación con aguas depuradas." y por el convenio con el Consorcio de la Costa Brava para la monitorización de la calidad de las aguas que circulan por el sistema de Aiguamolls de l'Empordà.

\section{BIBLIOGRAFÍA}

AGUESSE, P. \& F. MARAZANOF. 1965. Les modifications des milieux aquatiques de Camargue au cours des 30 dernières années. Annales de Limnologie, 1(2): 163-190.

AMANIEU, M., P. LEGENDRE, M. TROUSSELLIER \& G. F. FRISONI. 1989. Le programme Écothau: théorie écologique et base de la modélisation. Oceanologica Acta, 12 (3): 189-199.

BACH, J. 1990. L'ambient hidrogeològic de la plana litoral de l'Alt Empordà. Tesis doctoral. Universitat Autònoma de Barcelona. 
BIGOT, L. \& F. MARAZANOF. 1965. Considérations sur l'écologie des invertébrés terrestres et aquatiques des marismas du Guadalquivir (Andalucia). Vie et Milieu, 16: 441-473.

CARRILLO, P., L. CRUZ-PIZARRO, R. MORALES \& P. SÁNCHEZ-CASTILLO. 1987. Cambios estacionales en las comunidades de fitoplancton y zooplancton de la albufera de Adra. Limnetica, 3: 243-254.

COMÍN, F. A. 1984. Características físicas, químicas y fitoplancton de las lagunas costeras Encañizada, Tancada y Buda (Delta del Ebro). Oecologia aquatica, 7: 79-157.

GOSÁLBEZ, J., J. SERRA \& E. VELASCO (eds.). 1994. Els sistemes naturals dels Aiguamolls de l'Empordà. Treb. Inst. Cat. Hist. Nat., 13. 479 pp.

HERVÉ, P. \& J. BRUSLÉ. 1981. L'étang de CanetSaint-Nazaire (P.O.). Ecologie générale et ichthyofaune. Vie et Milieu, 31 (1): 17-25.

HEURTEAUX, P. 1992. Modifications du règime hydrique et salin des étangs du systéme de Vaccarès (Camargue, France) liées aux perturbations anthropiques des cinquante derniéres années. Annls. Limnol., 28 (2): 157-174.

JAMBU, M. 1991. Exploratory and Multivariate Data Analysis. Academic Press, Boston.

LASSERE, G. 1989. Biologie halieutique des lagunes. Ann. Biol., 28 (3): 161-186.

LÓPEZ, P. 1983. Aguas salinas epicontinentales próximas a la costa mediterránea española. Estudio del medio. Tesis doctoral. Universidad de Barcelona.

LÓPEZ, T., TOJA, J. \& GABELLONE, N.A., 1991. Limnological comparison of two peridunar ponds in the Doñana national park (Spain). Arch. Hydrobiol., 120(3): 357-378.

MARGALEF, R., 1983. Limnología. Omega, Barcelona.

MENÉNDEZ, M. \& F. A. COMÍN. 1986. Variación estacional del zooplancton en las lagunas costeras del Delta del Ebro (N.E. España). Oecologia aquatica, 8: 47-50.

MIRACLE, M. R. 1984. Heterogeneidad espacial de las comunidades fitoplanctónicas de la Albufera de Valencia. Limnetica, 1: 20-31.

MORENO-AMICH, R., X. QUINTANA, L. SUÑER, R. TROBAJO, S.GASCÓN, R. BARRERA, J. GIFRE \& M. MARTINOY. 1998. Seguiment de la qualitat de les aigües a la zona del Parc Natural dels aiguamolls de l'Empordà. In: Consorcio de la Costa Brava. CD-ROM Compendio de informes correspondientes al "Seguimiento de la calidad de les aguas a la zona del Parque Natural de Aiguamolls de l'Empordà." Consorcio de la Costa Brava, Girona.

PRAT, N., M. A.PUIG \& G. GONZÁLEZ. 1983. Predicció $\mathrm{i}$ control de la qualitat de les aigües dels rius Besòs i Llobregat, II. Estudis $i$ monografies del Servei del Medi Ambient, 9. Diputació de Barcelona. Barcelona.

PRETUS, J. 1985. Limnologia de l'Albufera d'Es Grau (Menorca). Aportacions a l'estudi del cicle anual. Tesi de Llicenciatura. Universitat de Barcelona.

PRETUS, J. L. 1989. Limnología de la Albufera de Menorca (Menorca, Spain). Limnetica, 5: 69-81.

QUINTANA, X. 1995. Fluctuacions a la maresma dels Aiguamolls de l'Empordà $i$ estructura de la comunitat biològica. Tesi doctoral. Universitat de Girona.

QUINTANA, X. \& F. A. COMÍN. 1989. Introducció a l'estudi limnològic de la llacuna del Ter Vell (Baix Empordà). Butll. Inst. Cat. Hist. Nat., 57 (Sec. Bot., 7): 23-34.

QUINTANA, X., F. A. COMÍN \& R. MORENOAMICH. 1998a. Nutrient and plankton dynamics in a mediterranean salt marsh dominated by incidents of flooding. Part 2: Response of the zooplankton community to disturbances. Journal of Plankton Research, 20(11): 2109-2127.

QUINTANA, X., R. MORENO-AMICH \& F. A. COMÍN. 1998b. Nutrient and plankton dynamics in a mediterranean salt marsh dominated by incidents of flooding. Part 1: Differential confinement of nutrients. Journal of Plankton Research, 20(11): 2089-2107.

ROS, J. D. 1987. El Mar Menor: fisiografía y biota acuática de una laguna hipersalina y su evolución reciente. En: Bases científicas para la protección de los humedales en España. 237-256. Real. Acad. Cienc. Exactas, Físicas y Naturales. Madrid.

SABATER, F. 1981. Comunidades de crustáceos de las lagunas de Tordera (Barcelona) en relación con sus características ambientales. Actas I Congreso Español de Limnología. Universidad de Barcelona.

SARGATAL, J. 1989. El Parc Natural: breu descripció geogràfica. In: Els Aiguamolls de l'Empordà. Aspectes ecològics, històrics $i$ socials. J. Y. Sargatal \& J. Fèlix (eds.): 11-18. Quaderns dels Indiketes núm. 3. ART-3 Carles Vallès editor. Figueres.

SARGATAL, J. \& J. FÈLIX. (eds.) 1989. Els Aiguamolls de l'Empordà. Aspectes ecològics, històrics $i$ socials. Quaderns dels Indiketes núm. 3. ART-3 Carles Vallès editor. Figueres. 